\title{
APPLICATIONS OF THE 3-DIM ICRH GLOBAL WAVE CODE FISIC AND COMPARISON WITH OTHER MODELS.
}

\author{
T. Krücken, M. Brambilla
}

IPP Garching, EURATOM Association, Fed. Rep. of Germany

INTRODUCTION. The code FISIC solves the integro-differential wave equations for h.f. waves in the ion cyclotron frequency range in the finite Larmor radius approximation in tokamak geometry. The equations are discretised by a semispectral approach, using cubic Hermite finite elements in the radial direction, and an expansion in Fourier modes in the poloidal direction. The latter allows the analytic evaluation of the orbit integrals along magnetic field lines in the presence of a poloidal component of the static magnetic field. The code is thus able to describe mode conversion to ion Bernstein waves, ion cyclotron damping at the fundamental and at the first harmonic, and electron transit time and Landau damping, in full toroidal geometry. A detailed description of the model and the numerical method will be given elsewhere $/ 1 /$. First results from FISIC were presented in $/ 2 /$. Since then a few improvements have been made, particularly in the description of collisional and electron damping.

Here we present two simulations of ICH in ASDEX $(R=167 \mathrm{~cm}, a=40 \mathrm{~cm}$, $B_{0}=2.24$ and $2.265 \mathrm{~T}, I_{p}=350$ and $380 \mathrm{kA}, n_{e}(0)=6 \cdot 10^{13}$; other parameters specified below), the first with low, the second with high single transit absorption according to simple estimates. For both cases we have run several toroidal modes, roughly scanning the spectrum excited by the antenna. We have used throughout between 80 and 100 radial elements, and 33 poloidal modes, ensuring good convergence within reasonable CPU time. The results are compared with those from two simpler codes, based on a slab model and on ray tracing, respectively. Agreement is good on the whole, but interesting purely toroidal effects are also identified, particularly on the efficiency of mode conversion and the propagation of ion Bernstein waves.

SECOND HARMONIC HEATING OF A $\mathrm{D}^{+}$PLASMA. The first example is a pure Deuterium plasma with $T_{D}=2 \mathrm{KeV}$ heated at the second harmonic cyclotron resonance $(f=33.5 \mathrm{Mhz})$. In this case we expect weak single pass absorption, good efficiency of mode conversion to IBW, and excitation of global eigenmodes. These are indeed clearly seen as peeks in the power spectrum scanning the toroidal modes (Fig. 1). Comparison with the slab-model calculations shows qualitative agreement, although matching of the individual peaks cannot be expected due to the different geometry. Fig. 2 shows contour plots of the real part of $E_{x}$ for one of the two resonant modes, $n_{\varphi}=14$. The pattern is dominated by the poloidal modes $m= \pm 1$ (as 
confirmed by examination of the poloidal spectrum). IBW are clearly visible to the high field side of the resonance, although for this mode their amplitude is small.

The total antenna resistance is found to be $R_{A}=2.36 \mathrm{Ohm}$, against $R_{A}=7.6 \mathrm{Ohm}$ in the slab model. The latter value is closer to the experimental one. The discrepancy might be due to the fact that the model of the antenna is more idealised in the fully toroidal code. Of the total power, $81.3 \%$ is absorbed by the ions, $4.4 \%$ by electron TTMP, $0.1 \%$ by ELD and $15 \%$ by damping of the IBW, which is a direct measure of mode conversion. In slab geometry model conversion to IBW is considerably more efficient $(62.2 \%)$, mainly due to modes with low $n_{\varphi}\left(\left|n_{\varphi}\right| \lesssim 5\right)$. This illustrates one essential difference between slab and fully toroidal simulations: in the latter even $n_{\varphi}=$ 0 corresponds to a quite broad spectrum of $k_{\|}$values, a feature which considerably reduces the efficiency of mode conversion.

SECOND HARMONIC HEATING OF $\mathrm{H}^{+}$IN A D ${ }^{+}$PLASMA. The second example is a two component plasma consisting of $25 \% \mathrm{H}$ and $75 \% \mathrm{D}$, preheated by neutral beam injection to central temperatures of $8.8 \mathrm{KeV}$ and $4.4 \mathrm{KeV}$, respectively. ICRH is applied at $\omega \approx 2 \Omega_{c H}(f=67 \mathrm{Mhz})$. In this case the single pass absorption is large even for low toroidal modes, suppressing mode conversion; therefore excitation of cavity eigenmodes is not expected. This is confirmed by the $n_{\varphi}$ power spectrum (Fig. 3), which shows almost no structure. Agreement with the slab simulation is even better than in the previous example, the major discrepancy occuring again for $n_{\varphi}=0$. In slab geometry this mode with $k_{\|}=0$ is very poorly damped (mainly by residual mode conversion); being non resonant, it is also poorly coupled. Broadening of the effective $k_{\|}$spectrum in the real toroidal geometry leads to much better damping by IC harmonic heating, so that the minimum in the power spectrum at $n_{\varphi}=0$ is nearly washed out. In the toroidal simulation therefore the radiated power is almost completely absorbed by harmonic ion cyclotron heating $(98.3 \%)$; the rest is absorbed by electron TTMP $(0.9 \%)$ and direct IBW damping $(0.8 \%)$. For the total antenna resistance we obtain $R_{A}=8.19 \mathrm{Ohm}$, compared to $R_{A}=10.2 \mathrm{Ohm}$ in the slab simulation.

Finally, Fig. 4 shows contour lines of the imaginary part of $E_{x}$ for $n_{\varphi}=16$. The large single transit absorption is evident here. Moreover, both the wave pattern and the power deposition profiles are in this case very similar to those obtained with ray tracing applying the code RAYIC /3/.

\section{REFERENCES.}

/1/ M. Brambilla, T. Krücken, submitted for publication.

/2/ M. Brambilla, T. Krücken, Proc. 14th Europ. Conf. on Controlled Fusion and Plasma Physics, Mardid 1987, Vol. 3, p. 996.

/3/ M. Brambilla, Comp. Phys. Reports 4 (1986) 1. 


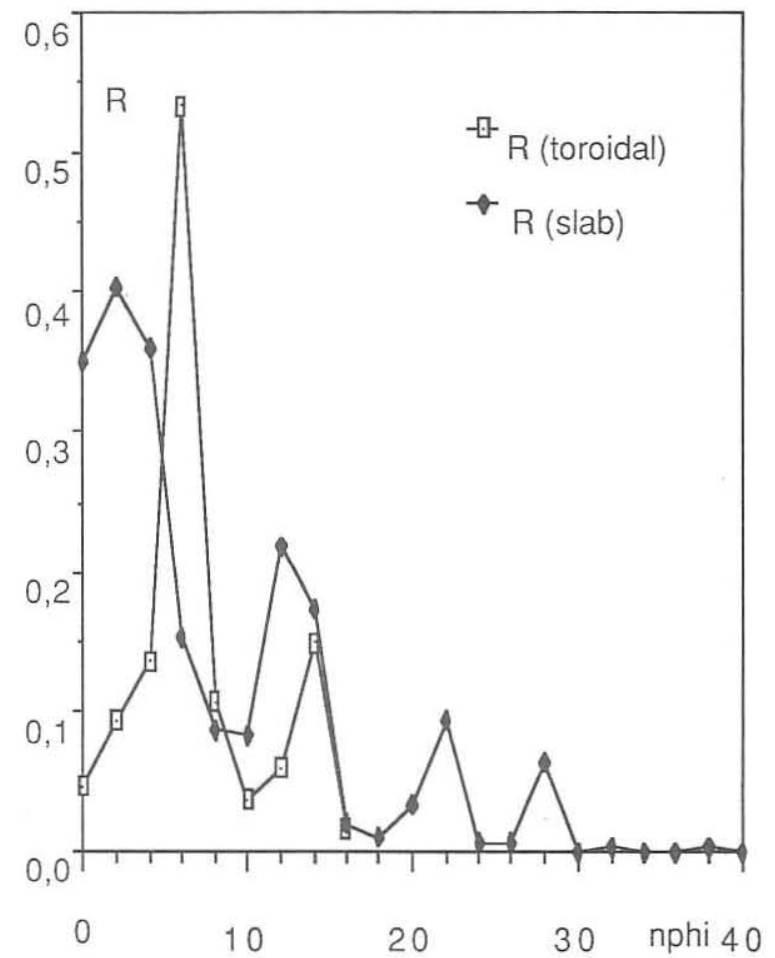

Fig. 1 Power spectrum. Harmonic heating of a Deuterium plasma, $\mathrm{T}(0)=2 \mathrm{keV}$.

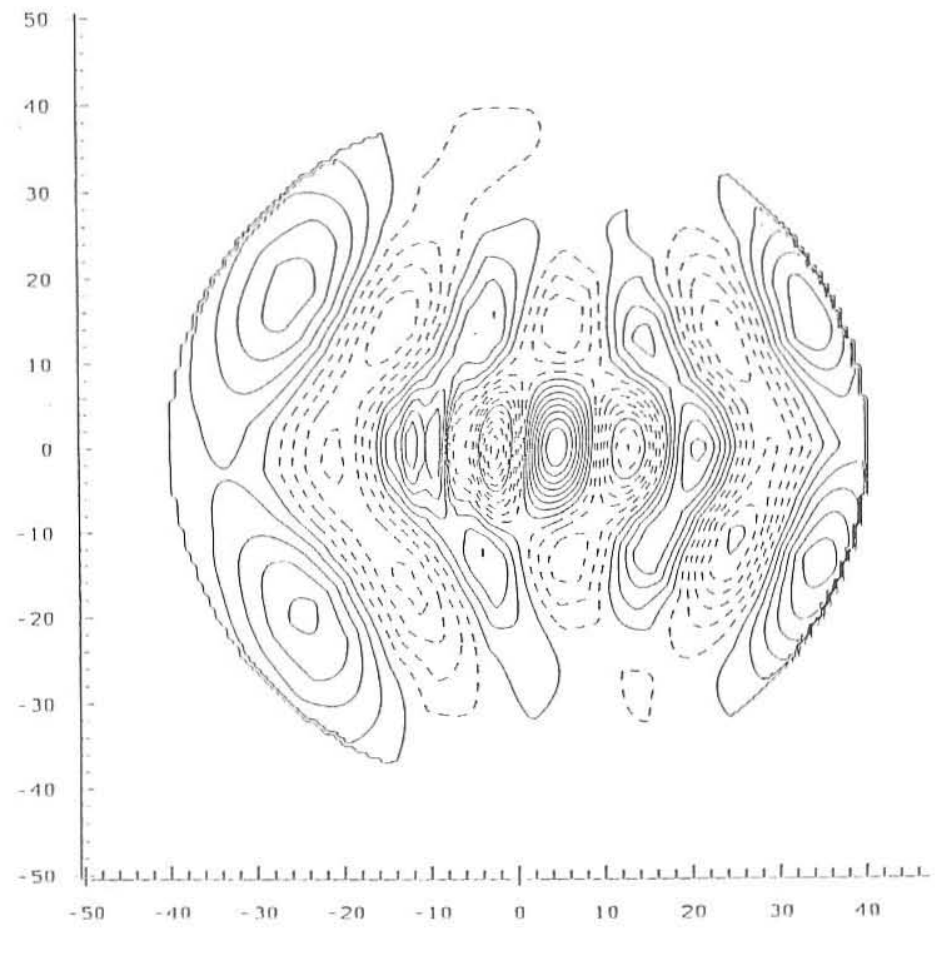

Fig. 2 - $\operatorname{Re}(E+)$ pattern in the poloidal crosssection for the resonant mode $n=14$. 

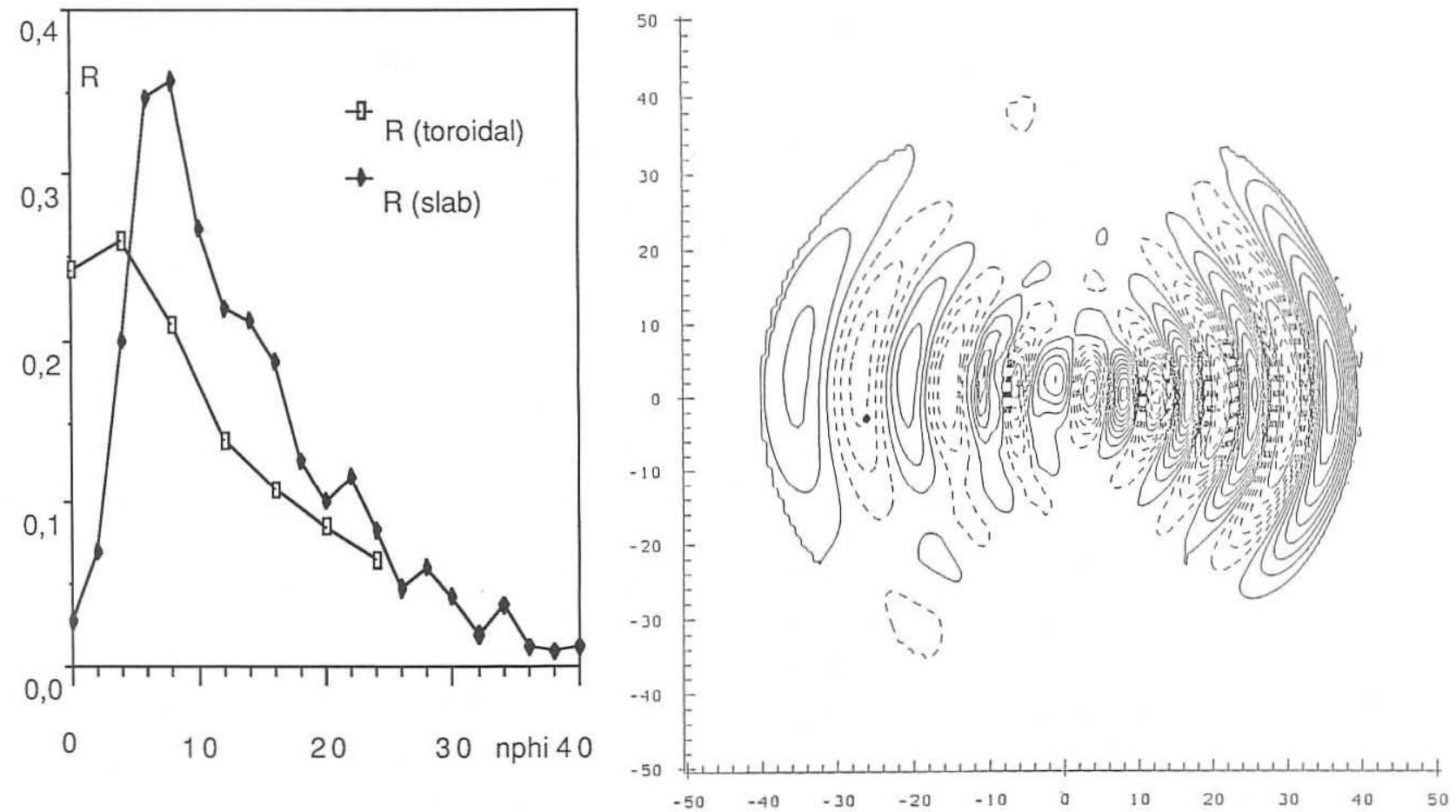

Fig. 3 - Power spectrum. Harmonic heating of $\mathrm{H}(25 \%, \mathrm{~T}(0)=8.8 \mathrm{keV})$ in $\mathrm{D}(75 \%, \mathrm{~T}(0)=4.4 \mathrm{keV})$
Fig. 4 - $\operatorname{Re}\left(E_{+}\right)$pattern in the poloidal crosssection, $n=16$. 